\title{
Catalyst free, one-pot, facile synthesis of novel pyrazolo-1,4-dihydropyridine derivative form pyranopyrazoles
}

\author{
Harvinder Singh Sohal a,*, Arun Goyal a, Rajshree Khare a, Kishanpal Singh b and Rajeev Sharma c \\ a Department of Chemistry, Maharishi Markandeshwar University, Mullana, 133207, Haryana, India \\ b Department of Chemistry, Punjabi University, Patiala, 147001, Punjab, India \\ c Department of Chemistry, Multani Mal Modi College, Patiala, 147001, Punjab, India \\ *Corresponding author at: Department of Chemistry, Maharishi Markandeshwar University, Mullana, 133207, Haryana, India. \\ Tel.: +91.988.8857705. Fax: +91.1731.274375. E-mail address: luckysohal.singh@gmail.com (H.S. Sohal).
}

\section{ARTICLE INFORMATION}

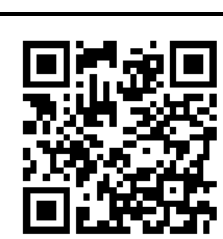

DOI: 10.5155 /eurjchem.5.2.227-232.967

Received: 11 November 2013

Received in revised form: 26 December 2013

Accepted: 28 December 2013

Online: 30 June 2014

\section{KEYWORDS}

Catalyst fre

Excellent yield

Acidic medium

Pyranopyrazoles

One pot synthesis

Pyrazolo-1,4-dihydropyridine

\section{Introduction}

Hantzsch condensation discovered 1,4-dihydropyridines (1,4-DHP's) in 1882 [1]. The 1,4-DHP's has attracted more attention, thanks to its presence in the coenzyme, diphosphopyridine nucleotide (DPNH) [2] and recognition as bio-active material. Many derivatives are commercialized in the market [3-7]. Fused 1,4-DHPs have also been able to make their presence felt largely due to ability to perform biological as well as pharmacological functions. 1,4-DHPs are an important class of $\mathrm{Ca}^{2+}$ channel blockers and are also known to be effective cardiovascular agents for the treatment of hypertension. Apart from these activities, DHPs are found to be as platelet-activity factor antagonists [8], calcium antagonists [9], antihypertensives [10], cerebral antischemic activity in the treatment of Alzheimer's disease and chemosensitizer acting in tumor therapy. Although the synthesis of pyrazolo-1,4dihydropyridines using expensive starting material like 3methyl-4,5-dihydro-1H-pyrazol-5-amine or 3-methyl-1-phenyl4,5-dihydro- $1 H$-pyrazol-5-amine, has considerably contributed to the development of new pyrazolo-1,4-DHPs [11-15], yet, the achieved molecule remains beyond the reach of most manufacturers, as it involves the usage of expensive starting material. The molecule also does not remain confined to the use of generally used methodologies and therefore, additional and exquisite methodologies need to be brought into use [14]

Therefore, recognizing the development of a clean, green and efficient procedure as the need of the hour, and persisting with our work on heterocyclics [16-18] and in continuation of our previous work (Scheme 1), we have been able to produce some pyrazolo-1,4-DHPs from easily available pyranopyrazoles (Figure 1) [16]. The present protocol is simpler as, use of harsh reaction condition scheme is not a pre-requisite to it (Scheme 2).

\section{Experimental}

\subsection{Instrumentation}

Materials were obtained from commercial suppliers and were used without further purifications. Melting points were recorded in open end capillaries and are uncorrected. ${ }^{1} \mathrm{H}$ and ${ }^{13} \mathrm{C}$ NMR spectra were recorded in DMSO- $d_{6}$ on a Bruker Avance II $400 \mathrm{MHz}$ spectrometer; chemical shifts $(\delta)$ are reported in ppm relative to TMS as internal standard. The mass spectrum and IR spectra were recorded at LC-MS Spectrometer Model QToF Micro Waters and Perkin-Elmer Spectrum II infrared spectrophotometer, respectively. 


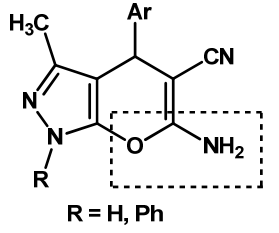

Pyranopyrazoles (Previous work)

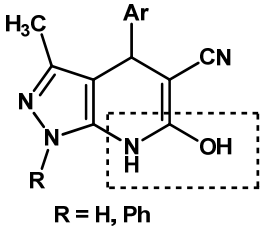

Pyrazolo-1,4-Dihydropyridines (Present work)

Scheme 1

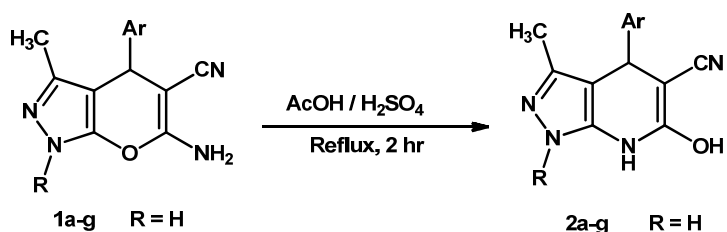

$\mathrm{Ar}=\mathrm{C}_{6} \mathrm{H}_{5}, 4-\mathrm{Cl}-\mathrm{C}_{6} \mathrm{H}_{4}, 2-\mathrm{Cl}-\mathrm{C}_{6} \mathrm{H}_{4}, 4-\mathrm{NO}_{2}-\mathrm{C}_{6} \mathrm{H}_{4}$, 2- $\mathrm{NO}_{2}-\mathrm{C}_{6} \mathrm{H}_{4}, 4-\mathrm{OMe}-\mathrm{C}_{6} \mathrm{H}_{4}, 4-\mathrm{Me}^{-\mathrm{C}_{6}} \mathrm{H}_{4}$

1h-n $\mathrm{R}=\mathrm{Ph}$

$\mathrm{Ar}=\mathrm{C}_{6} \mathrm{H}_{5}, 4-\mathrm{Cl}-\mathrm{C}_{6} \mathrm{H}_{4}, 2-\mathrm{Cl}-\mathrm{C}_{6} \mathrm{H}_{4}, 4-\mathrm{NO}_{2}-\mathrm{C}_{6} \mathrm{H}_{4}$ $2-\mathrm{NO}_{2}-\mathrm{C}_{6} \mathrm{H}_{4}, 4-\mathrm{OMe}-\mathrm{C}_{6} \mathrm{H}_{4}, 4-\mathrm{Me}-\mathrm{C}_{6} \mathrm{H}_{4}$
$\mathrm{Ar}=\mathrm{C}_{6} \mathrm{H}_{5}, 4-\mathrm{Cl}-\mathrm{C}_{6} \mathrm{H}_{4}, 2-\mathrm{Cl}-\mathrm{C}_{6} \mathrm{H}_{4}, 4-\mathrm{NO}_{2}-\mathrm{C}_{6} \mathrm{H}_{4}$, 2- $\mathrm{NO}_{2}-\mathrm{C}_{6} \mathrm{H}_{4}, 4-\mathrm{OMe}-\mathrm{C}_{6} \mathrm{H}_{4}, 4-\mathrm{Me}-\mathrm{C}_{6} \mathrm{H}_{4}$

2h-n $\quad R=P h$

$\mathrm{Ar}=\mathrm{C}_{6} \mathrm{H}_{5}, 4-\mathrm{Cl}-\mathrm{C}_{6} \mathrm{H}_{4}, 2-\mathrm{Cl}-\mathrm{C}_{6} \mathrm{H}_{4}, 4-\mathrm{NO}_{2}-\mathrm{C}_{6} \mathrm{H}_{4}$

$2-\mathrm{NO}_{2}-\mathrm{C}_{6} \mathrm{H}_{4}, 4-\mathrm{OMe}-\mathrm{C}_{6} \mathrm{H}_{4}, 4-\mathrm{Me}-\mathrm{C}_{6} \mathrm{H}_{4}$

Scheme 2

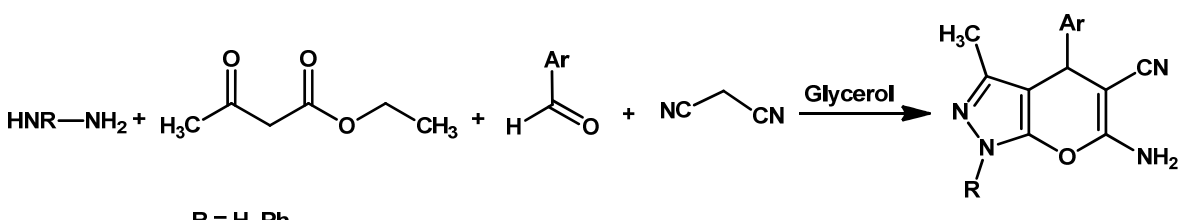

$\mathrm{R}=\mathrm{H}, \mathrm{Ph}$

$\mathrm{Ar}=\mathrm{C}_{6} \mathrm{H}_{5}, 4-\mathrm{Cl}-\mathrm{C}_{6} \mathrm{H}_{4}, 2-\mathrm{Cl}-\mathrm{C}_{6} \mathrm{H}_{4}, 4-\mathrm{NO}_{2}-\mathrm{C}_{6} \mathrm{H}_{4}, 2-\mathrm{NO}_{2}-\mathrm{C}_{6} \mathrm{H}_{4}, 4-\mathrm{OMe}-\mathrm{C}_{6} \mathrm{H}_{4}, 4-\mathrm{Me}_{-}-\mathrm{C}_{6} \mathrm{H}_{4}$

Figure 1. Synthesis of pyranopyrazoles using glycerol as green solvent.

Elemental analyses (C, H, and N) were performed using a Thermo Scientific elemental analyzer.

\subsection{Synthesis}

Pyranopyrazoles (1a-n) are prepared by using the given literature procedure [16]. In a conical flask, hydrazine hydrate/phenyl hydrazine $(10 \mathrm{mmol})$, ethylacetoacetate $(10$ $\mathrm{mmol})$, aromatic aldehyde $(10 \mathrm{mmol})$ and malononitrile were added successively in glycerol $(20 \mathrm{~mL})$. Reaction mixture was stirred at $80^{\circ} \mathrm{C}$. After the completion of reaction (monitored by TLC), diluted the reaction mixture with ice cold water. Filtered the solid thus obtained and recrystallized with ethanol to afford compound 1a-n.

6-Hydroxy-3-methyl-4-phenyl-4,7-dihydro-1H-pyrazolo[3,4b]pyridine-5-carbonitrile (2a): In a conical flask pyranopyrazole $(1 \mathrm{mmol})$ was taken in the mixture of acetic acid $(3 \mathrm{~mL})$ and sulphuric acid $(0.1 \mathrm{~mL})$ and reflux at $110^{\circ} \mathrm{C}$ for the stipulated time Table 1. After the completion of reaction (vide TLC), reaction mixture was cooled to room temperature, solid separated out. Filtered and dried, recrystallized from ethanol to afford compound 2a (Entry 1, Table 2). Yield: 88\%. M.p.: >300 ${ }^{\circ} \mathrm{C}$. FT-IR (KBr, v, cm-1): $3532 v(\mathrm{O}-\mathrm{H}$ Str.), $3460 v(\mathrm{~N}-\mathrm{H}$ Str.), $3390 v$ (N-H Str.), $2206 v$ (C $\equiv N$ Str.). ${ }^{1} \mathrm{H}$ NMR $(400 \mathrm{MHz}$, DMSO$\left.d_{6}, \delta, \mathrm{ppm}\right): 1.78\left(\mathrm{~s}, 3 \mathrm{H}, \mathrm{CH}_{3}\right), 4.53(\mathrm{~s}, 1 \mathrm{H}, \mathrm{CH}), 7.11-7.81(\mathrm{~m}, 5 \mathrm{H}$, $\mathrm{Ar}-\mathrm{H}), 9.82(\mathrm{~s}, 1 \mathrm{H}, \mathrm{OH}), 12.14(\mathrm{~s}, 1 \mathrm{H}, \mathrm{NH}), 13.15(\mathrm{~s}, 1 \mathrm{H}, \mathrm{NH}) .{ }^{13} \mathrm{C}$ NMR (100 MHz, DMSO- $\left.d_{6}, \delta, \mathrm{ppm}\right): 160.2,156.9,148.1,139.5$, $135.4,122.1,120.1,114.1,96.7,77.9,56.6,52.6,34.3,9.7$. MS (EI, $m / z(\%)): 253\left(\mathrm{M}^{+}, 12\right)$. Anal. calcd. for $\mathrm{C}_{14} \mathrm{H}_{12} \mathrm{~N}_{4} \mathrm{O}$ : C, 66.65; H, 4.79; N, 22.21. Found: C, 66.61; H, 4.78; N, 22.19\%.
Similarly, other pyranopyrazoles $\mathbf{1 b}-\mathbf{n}$ were reacted to afford various pyrazolo-1,4-dihydropyridines derivatives $\mathbf{2 b}$-n (Table 2). Data obtained using advanced spectral techniques for some selected compounds have been summarized.

6-Hydroxy-3-methyl-4-(4-chlorophenyl)-4,7-dihydro-1Hpyrazolo[3,4-b]pyridine-5-carbonitrile (2b): Yield: 84\%. M.p.: $>300{ }^{\circ} \mathrm{C}$. FT-IR (KBr, $\left.v, \mathrm{~cm}^{-1}\right): 3540 v(\mathrm{O}-\mathrm{H}$ Str.), $3510 v(\mathrm{~N}-\mathrm{H}$ Str.), $3442 v\left(\mathrm{~N}-\mathrm{H}\right.$ Str.), $2264 v\left(\mathrm{C} \equiv \mathrm{N}\right.$ Str.). ${ }^{1} \mathrm{H}$ NMR $(400 \mathrm{MHz}$, DMSO- $\left.d_{6}, \delta, \mathrm{ppm}\right): 1.73\left(\mathrm{~s}, 3 \mathrm{H}, \mathrm{CH}_{3}\right), 4.51(\mathrm{~s}, 1 \mathrm{H}, \mathrm{CH}), 6.91-7.45$ (m, 4H, Ar-H), 9.85 (s, 1H, OH), $12.01(\mathrm{~s}, 1 \mathrm{H}, \mathrm{NH}), 13.11(\mathrm{~s}, 1 \mathrm{H}$, NH). ${ }^{13} \mathrm{C}$ NMR (100 MHz, DMSO- $\left.d_{6}, \delta, \mathrm{ppm}\right): 162.1,153.3,149.7$, 144.2, 136.7, 133.0, 125.3, 120.9, 118.6, 98.3, 77.5, 53.2, 35.7, 9.7. MS (EI, $m / z(\%)$ ): $288\left(\mathrm{M}^{+}, 10\right)$. Anal. calcd. for $\mathrm{C}_{14} \mathrm{H}_{11} \mathrm{ClN}_{4} \mathrm{O}$ : C, 58.65; H, 3.87; N, 19.54. Found: C, 58.63; H, 3.84; N, 19.53\%.

6-Hydroxy-3-methyl-4-(2-nitrophenyl)-4,7-dihydro-1Hpyrazolo[3,4-b]pyridine-5-carbonitrile (2e): Yield: 85\%. M.p.: $>300{ }^{\circ} \mathrm{C}$. FT-IR (KBr, v, cm-1): $3572 v(\mathrm{O}-\mathrm{H}$ Str.), $3520 v(\mathrm{~N}-\mathrm{H}$ Str.), $3450 v$ (N-H Str.), $2340 v\left(\mathrm{C} \equiv \mathrm{N}\right.$ Str.). ${ }^{1} \mathrm{H}$ NMR $(400 \mathrm{MHz}$, DMSO- $\left.d_{6}, \delta, \mathrm{ppm}\right): 1.79\left(\mathrm{~s}, 3 \mathrm{H}, \mathrm{CH}_{3}\right), 4.76(\mathrm{~s}, 1 \mathrm{H}, \mathrm{CH}), 7.60-8.06$ (m, 4H, Ar-H), 10.01 (s, 1H, OH), 12.19 (s, 1H, NH), 13.21 (s, 1H, $\mathrm{NH}) .{ }^{13} \mathrm{C}$ NMR $\left(100 \mathrm{MHz}\right.$, DMSO- $\left.d_{6}, \delta, \mathrm{ppm}\right): 161.0,154.7,147.8$, 146.5, 135.6, 134.0, 129.6, 121.7, 120.3, 96.3, 78.5, 56.4, 39.5 9.7. MS (EI, $m / z(\%)): 298\left(\mathrm{M}^{+}, 13\right)$. Anal. calcd. for $\mathrm{C}_{4} \mathrm{H}_{11} \mathrm{~N}_{5} \mathrm{O}_{3}$ : C, 56.56; H, 3.73; N, 23.56. Found: C, 56.53; H, 3.69; N, 23.54\%.

6-Hydroxy-3-methyl-4-(4-methoxyphenyl)-4,7-dihydro-1Hpyrazolo[3,4-b]pyridine-5-carbonitrile (2f): Yield: 81\%. M.p.: 290-292 ${ }^{\circ} \mathrm{C}$. FT-IR (KBr, $\left.v, \mathrm{~cm}^{-1}\right): 3510 v(\mathrm{O}-\mathrm{H}$ Str.), $3490 v(\mathrm{~N}-\mathrm{H}$ Str.), $3460 v\left(\mathrm{~N}-\mathrm{H}\right.$ Str.), $2252 v\left(\mathrm{C} \equiv \mathrm{N}\right.$ Str.). ${ }^{1} \mathrm{H}$ NMR $(400 \mathrm{MHz}$, DMSO- $\left.d_{6}, \delta, \mathrm{ppm}\right): 1.72\left(\mathrm{~s}, 3 \mathrm{H}, \mathrm{CH}_{3}\right), 3.68\left(\mathrm{~s}, 3 \mathrm{H}, \mathrm{OCH}_{3}\right), 4.47(\mathrm{~s}$, $1 \mathrm{H}, \mathrm{CH}), 7.21-7.80(\mathrm{~m}, 4 \mathrm{H}, \mathrm{Ar}-\mathrm{H}), 9.92(\mathrm{~s}, 1 \mathrm{H}, \mathrm{OH}), 12.08(\mathrm{~s}, 1 \mathrm{H}$ $\mathrm{NH}), 13.06(\mathrm{~s}, 1 \mathrm{H}, \mathrm{NH})$. 
Table 1.Effect of temperature on the synthesis of compound 2a.

\begin{tabular}{lllll}
\hline Entry & Compound & Temperature $\left.\mathbf{~}^{\circ} \mathbf{C}\right)$ & Time (h) & Yield a $(\%)$ \\
\hline 1 & $2 \mathrm{a}$ & 70 & 9 & 20 \\
2 & $2 \mathrm{a}$ & 80 & 4 & 52 \\
3 & $2 \mathrm{a}$ & 90 & 3 & 70 \\
4 & $2 \mathrm{a}$ & 100 & 2 & 79 \\
5 & $2 \mathrm{a}$ & 110 & 2 & 88 \\
6 & $2 \mathrm{a}$ & $120^{\mathrm{b}}$ & 2 & 87 \\
7 & $2 \mathrm{a}$ & $130 \mathrm{~b}$ & & 82 \\
\hline a Yield refer to combined amounts of different crops.
\end{tabular}

a Yield refer to combined amounts of different crops.

b Reaction were carried out in silicon oil bath with the use of cold water in condenser.

${ }^{13} \mathrm{C}$ NMR $\left(100 \mathrm{MHz}, \mathrm{DMSO}-d_{6}, \delta, \mathrm{ppm}\right): 160.5,157.9,154.7$, 136.1, 135.4, 128.3, 120.7, 113.4, 97.6, 78.5, 57.9, 54.7, 39.5, 35.6, 9.7. MS (EI, $m / z(\%)): 283\left(\mathrm{M}^{+}, 09\right)$. Anal. calcd. for $\mathrm{C}_{15} \mathrm{H}_{14} \mathrm{~N}_{4} \mathrm{O}_{2}$ : C, 63.82; H, 5.00; N, 11.34. Found: C, 63.79; H, 4.98; $\mathrm{N}, 11.33 \%$.

6-Hydroxy-3-methyl-4-(4-methylphenyl)-4,7-dihydro-1Hpyrazolo[3,4-b]pyridine-5-carbonitrile (2g): Yield: 82\%. M.p.: $>300{ }^{\circ} \mathrm{C}$. FT-IR (KBr, v, cm-1): 3501 v(O-H Str.), $3489 v(\mathrm{~N}-\mathrm{H}$ Str.), $3472 v\left(\mathrm{~N}-\mathrm{H}\right.$ Str.), $2199 v\left(\mathrm{C} \equiv \mathrm{N}\right.$ Str.). ${ }^{1} \mathrm{H}$ NMR (400 MHz, DMSO- $\left.d_{6}, \delta, \mathrm{ppm}\right): 1.75\left(\mathrm{~s}, 3 \mathrm{H}, \mathrm{CH}_{3}\right), 2.21\left(\mathrm{~s}, 3 \mathrm{H}, \mathrm{CH}_{3}\right) 4.46(\mathrm{~s}$, 1H, CH), 7.01-7.42 (m, 4H, Ar-H), 9.72 (s, 1H, OH), 11.98 (s, 1H, $\mathrm{NH}), 12.95$ (s, $1 \mathrm{H}, \mathrm{NH}) .{ }^{13} \mathrm{C}$ NMR (100 MHz, DMSO- $\left.d_{6}, \delta, \mathrm{ppm}\right)$ : 159.0, 156.4, 150.2, 133.7, 130.8, 124.1, 123.0, 116.5, 95.2, 78.6, 56.4, 51.2, 40.2, 24.3, 9.7. MS (EI, $m / z(\%)): 267\left(\mathrm{M}^{+}, 10\right)$. Anal. calcd. for $\mathrm{C}_{15} \mathrm{H}_{14} \mathrm{~N}_{4} \mathrm{O}$ : C, 67.65; H, 5.30; N, 21.04. Found: C, 67.63; $\mathrm{H}, 5.28 ; \mathrm{N}, 21.01 \%$.

6-Hydroxy-3-methyl-1,4-diphenyl-4,7-dihydro-1H-pyrazolo [3,4-b]pyridine-5-carbonitrile (2h): Yield: 85\%. M.p.: 228-230 ${ }^{\circ} \mathrm{C}$. FT-IR (KBr, v, cm${ }^{-1}$ ): $3490 v(\mathrm{O}-\mathrm{H}$ Str.), $3430 v(\mathrm{~N}-\mathrm{H}$ Str.), $2221 v\left(\mathrm{C} \equiv \mathrm{N}\right.$ Str.). ${ }^{1} \mathrm{H}$ NMR (400 MHz, DMSO- $\left.d_{6}, \delta, \mathrm{ppm}\right): 1.90$ (s, $\left.3 \mathrm{H}, \mathrm{CH}_{3}\right), 4.57(\mathrm{~s}, 1 \mathrm{H}, \mathrm{CH}), 7.21-7.48(\mathrm{~m}, 10 \mathrm{H}, \mathrm{Ar}-\mathrm{H}), 10.07$ (s, $1 \mathrm{H}, \mathrm{OH}), 13.25$ (s, $1 \mathrm{H}, \mathrm{NH}) .{ }^{13} \mathrm{C}$ NMR (100 MHz, DMSO-d6, $\delta$, ppm): 159.2, 144.2, 143.3, 141.2, 138.8, 135.1, 128.6, 123.4, 120.5, 113.4, 95.5, 77.5, 74.1, 58.2, 40.6, 39.3, 38.1, 36.6, 33.6, 12.4. MS (EI, $m / z(\%)): 329\left(\mathrm{M}^{+}, 13\right)$. Anal. calcd. for $\mathrm{C}_{20} \mathrm{H}_{16} \mathrm{~N}_{4} \mathrm{O}$ : C, 73.15; H, 4.91; N, 17.06. Found: C, 73.12; H, 4.89; N, 17.04\%.

6-Hydroxy-3-methyl-4-(4-chlorophenyl)-1-phenyl-4,7dihydro-1H-pyrazolo[3,4-b]pyridine-5-carbonitrile (2i): Yield: 82\%. M.p.: 250-252 ${ }^{\circ} \mathrm{C}$. FT-IR (KBr, v, cm-1): 3499 v(O-H Str.), $3434 v\left(\mathrm{~N}-\mathrm{H}\right.$ Str.), $2230 v\left(\mathrm{C} \equiv \mathrm{N}\right.$ Str.). ${ }^{1} \mathrm{H}$ NMR (400 MHz, DMSO$\left.d_{6}, \delta, \mathrm{ppm}\right): 1.81\left(\mathrm{~s}, 3 \mathrm{H}, \mathrm{CH}_{3}\right), 4.49(\mathrm{~s}, 1 \mathrm{H}, \mathrm{CH}), 7.10-7.62(\mathrm{~m}, 9 \mathrm{H}$, Ar-H), 10.02 (s, 1H, OH), 13.29 (s, 1H, NH). ${ }^{13} \mathrm{C}$ NMR (100 MHz, DMSO- $\left.d_{6}, \delta, \mathrm{ppm}\right): 159.3,147.2,144.7,142.2,138.2,137.1$, 129.7, 126.4, 122.6, 116.4, 97.4, 79.7, 78.1, 59.0, 41.3, 39.7, 39.1, 38.4, 35.6, 12.6. MS (EI, $m / z(\%)): 363\left(\mathrm{M}^{+}, 10\right)$. Anal. calcd. for $\mathrm{C}_{20} \mathrm{H}_{15} \mathrm{ClN}_{4} \mathrm{O}$ : C, 66.21; H, 4.17; N, 15.44. Found: C, $66.19 ; \mathrm{H}, 4.16 ; \mathrm{N}, 15.41 \%$.

6-Hydroxy-3-methyl-4-(2-chlorophenyl)-1-phenyl-4,7dihydro-1H-pyrazolo[3,4-b]pyridine-5-carbonitrile (2j): Yield: 81\%. M.p.: 220-222 C. FT-IR (KBr, v, cm-1): 3498 v(O-H Str.), $3480 v\left(\mathrm{~N}-\mathrm{H}\right.$ Str.), $2229 v\left(\mathrm{C} \equiv \mathrm{N}\right.$ Str.). ${ }^{1} \mathrm{H}$ NMR (400 MHz, DMSO$\left.d_{6}, \delta, \mathrm{ppm}\right): 1.87\left(\mathrm{~s}, 3 \mathrm{H}, \mathrm{CH}_{3}\right), 4.51(\mathrm{~s}, 1 \mathrm{H}, \mathrm{CH}), 7.11-7.69(\mathrm{~m}, 9 \mathrm{H}$, Ar-H), 10.06 (s, 1H, OH), 13.27 (s, 1H, NH). ${ }^{13} \mathrm{C}$ NMR (100 MHz, DMSO- $\left.d_{6}, \delta, \mathrm{ppm}\right): 159.3,147.5,144.1,143.0,138.2,136.9$, $129.7,125.8,124.0,118.4,96.9,78.9,77.5,58.9,45.3,40.7$, 39.9, 38.1, 36.5, 12.6. MS (EI, $m / z(\%)): 363\left(\mathrm{M}^{+}, 09\right)$. Anal. calcd. for $\mathrm{C}_{20} \mathrm{H}_{15} \mathrm{ClN}_{4} \mathrm{O}$ : C, 66.21; H, 4.17; N, 15.44. Found: C, 66.20; H, 4.15; N, $15.42 \%$.

6-Hydroxy-3-methyl-4-(4-nitrophenyl)-1-phenyl-4,7-dihydro1H-pyrazolo[3,4-b]pyridine-5-carbonitrile (2k): Yield: 88\%. M.p.: 292-294 ${ }^{\circ} \mathrm{C}$. FT-IR (KBr, v, cm-1): $3560 v(\mathrm{O}-\mathrm{H}$ Str.), 3499 $v\left(\mathrm{~N}-\mathrm{H}\right.$ Str.), $2290 v\left(\mathrm{C} \equiv \mathrm{N}\right.$ Str.). ${ }^{1} \mathrm{H}$ NMR (400 MHz, DMSO- $d_{6}, \delta$, ppm): $1.74\left(\mathrm{~s}, 3 \mathrm{H}, \mathrm{CH}_{3}\right), 4.90(\mathrm{~s}, 1 \mathrm{H}, \mathrm{CH}), 7.37-8.41(\mathrm{~m}, 9 \mathrm{H}, \mathrm{Ar}-$ $\mathrm{H}), 10.21(\mathrm{~s}, 1 \mathrm{H}, \mathrm{OH}), 13.36(\mathrm{~s}, 1 \mathrm{H}, \mathrm{NH}) .{ }^{13} \mathrm{C}$ NMR $(100 \mathrm{MHz}$, DMSO- $\left.d_{6}, \delta, \mathrm{ppm}\right): 159.6,147.9,145.7,145.0,143.9,137.4$, $134.4,129.9,129.0,125.9,122.1,119.9,97.4,78.3,57.2,40.1$, 39.3, 38.9, 36.4, 12.8. MS (EI, $m / z(\%)): 374\left(\mathrm{M}^{+}, 12\right)$. Anal. calcd. for $\mathrm{C}_{20} \mathrm{H}_{15} \mathrm{~N}_{5} \mathrm{O}_{3}$ : C, 64.34; $\mathrm{H}, 4.05 ; \mathrm{N}, 18.76$. Found: C, 64.33; H, 4.04; N, 18.76\%.

6-Hydroxy-3-methyl-4-(2-nitrophenyl)-1-phenyl-4,7-dihydro1H-pyrazolo[3,4-b]pyridine-5-carbonitrile (2l): Yield: 83\%. M.p.: 286-288 ${ }^{\circ} \mathrm{C}$. FT-IR (KBr, v, cm$\left.{ }^{-1}\right): 3559 v(\mathrm{O}-\mathrm{H}$ Str.), $3440 v(\mathrm{~N}-\mathrm{H}$ Str.), $2263 v\left(\mathrm{C} \equiv \mathrm{N}\right.$ Str.). ${ }^{1} \mathrm{H}$ NMR (400 MHz, DMSO- $\left.d_{6}, \delta, \mathrm{ppm}\right)$ : $1.80\left(\mathrm{~s}, 3 \mathrm{H}, \mathrm{CH}_{3}\right), 4.87(\mathrm{~s}, 1 \mathrm{H}, \mathrm{CH}), 7.32-8.11(\mathrm{~m}, 9 \mathrm{H}, \mathrm{Ar}-\mathrm{H})$, $10.20(\mathrm{~s}, 1 \mathrm{H}, \mathrm{OH}), 13.27$ (s, $1 \mathrm{H}, \mathrm{NH}) .{ }^{13} \mathrm{C}$ NMR (100 MHz, DMSO$\left.d_{6}, \delta, \mathrm{ppm}\right): 159.6,147.8,145.5,144.8,143.5,136.9,134.0$, 130.2 , 129.1, 124.6, 121.9, 119.7, 98.0, 78.1, 55.1, 41.2, 39.0, 37.9, 36.0, 12.7. MS (EI, $m / z(\%)): 374\left(\mathrm{M}^{+}, 14\right)$. Anal. calcd. for $\mathrm{C}_{20} \mathrm{H}_{15} \mathrm{~N}_{5} \mathrm{O}_{3}$ : C, 64.34; H, 4.05; N, 18.76. Found: C, 64.31; H, 4.00; $\mathrm{N}, 18.75 \%$.

6-Hydroxy-3-methyl-4-(4-methoxyphenyl)-1-phenyl-4,7dihydro-1H-pyrazolo[3,4-b]pyridine-5-carbonitrile (2m): Yield: 81\%. M.p.: $265-267^{\circ} \mathrm{C}$. FT-IR (KBr, v, cm$\left.{ }^{-1}\right)$ : $3520 v(\mathrm{O}-\mathrm{H}$ Str.) $3425 v\left(\mathrm{~N}-\mathrm{H}\right.$ Str.), $2219 v\left(\mathrm{C} \equiv \mathrm{N}\right.$ Str.). ${ }^{1} \mathrm{H}$ NMR (400 MHz, DMSO$\left.d_{6}, \delta, \mathrm{ppm}\right): 1.81\left(\mathrm{~s}, 3 \mathrm{H}, \mathrm{CH}_{3}\right), 3.71\left(\mathrm{~s}, 3 \mathrm{H}, \mathrm{OCH}_{3}\right), 4.56(\mathrm{~s}, 1 \mathrm{H}$ $\mathrm{CH})$, 6.91-7.93 (m, 9H, Ar-H), $10.18(\mathrm{~s}, 1 \mathrm{H}, \mathrm{OH}), 13.17$ (s, 1H, NH). ${ }^{13}$ C NMR (100 MHz, DMSO- $\left.d_{6}, \delta, p p m\right): 159.1,158.1,145.2$, 143.7, 137.6, 135.3, 128.9, 125.7, 119.8, 113.5, 99.4, 98.5, 78.5, 58.8, 54.8, 40.2, 39.8, 39.1, 38.2, 30.5, 12.5. MS (EI, $m / z(\%)$ ): $359\left(\mathrm{M}^{+}, 08\right)$. Anal. calcd. for $\mathrm{C}_{21} \mathrm{H}_{18} \mathrm{~N}_{4} \mathrm{O}_{2}$ : C, 70.38; $\mathrm{H}, 5.06$; $\mathrm{N}$, 15.63. Found: C, 70.35; H, 5.05; N, $15.61 \%$.

6-Hydroxy-3-methyl-4-(4-methylphenyl)-1-phenyl-4,7dihydro-1H-pyrazolo[3,4-b]pyridine-5-carbonitrile (2n): Yield: 80\%. M.p.:287-288 'C. FT-IR (KBr, v, cm-1): 3506 v(O-H Str.), $3419 v\left(\mathrm{~N}-\mathrm{H}\right.$ Str.), $2209 v\left(\mathrm{C} \equiv \mathrm{N}\right.$ Str.). ${ }^{1} \mathrm{H}$ NMR (400 MHz, DMSO$\left.d_{6}, \delta, \mathrm{ppm}\right): 1.79\left(\mathrm{~s}, 3 \mathrm{H}, \mathrm{CH}_{3}\right), 2.61\left(\mathrm{~s}, 3 \mathrm{H}, \mathrm{CH}_{3}\right), 4.53(\mathrm{~s}, 1 \mathrm{H}, \mathrm{CH})$, 7.41-8.24 (m, 9H, Ar-H), 10.09 (s, 1H, OH), 12.99 (s, 1H, NH). ${ }^{13} \mathrm{C}$ NMR (100 MHz, DMSO- $\left.d_{6}, \delta, \mathrm{ppm}\right): 159.2,145.2,143.7,140.2$, $137.5,136.0,128.8,127.4,125.6,119.8,98.4,78.7,78.3,58.9$, $40.2,39.8,39.4,38.9,36.6,20.6,12.5$. MS (EI, $m / z(\%)): 343$ $\left(\mathrm{M}^{+}, 11\right)$. Anal. calcd. for $\mathrm{C}_{21} \mathrm{H}_{18} \mathrm{~N}_{4} \mathrm{O}$ : C, 73.67; $\mathrm{H}, 5.30 ; \mathrm{N}, 16.36$. Found: C, 73.66; H, 5.28; N, 16.36\%.

\section{Result and discussion}

Reactions of pyranopyrazole 1a with the mixture acetic acid and sulphuric acid were carried out at different temperatures $\left(70-130{ }^{\circ} \mathrm{C}\right)$. It was observed that $110^{\circ} \mathrm{C}$ is the optimal temperature for the synthesis of pyrazolo-1,4dihydropyridines. Further, rise in temperature results in the decomposition of the reaction mixture (Table 1).

The structure of the compound 2a was confirmed with the use of spectral techniques. In IR spectrum absorption at 3532 $\mathrm{cm}^{-1}$ represents the $\mathrm{O}-\mathrm{H}$ stretching, absorption at 3460 and $3390 \mathrm{~cm}^{-1}$ for two $\mathrm{N}-\mathrm{H}$ stretching, a sharp absorption peak at $2206 \mathrm{~cm}^{-1}$ represent $\mathrm{C} \equiv \mathrm{N}$ stretching. In ${ }^{1} \mathrm{H}$ NMR spectra peaks two singletat 13.15 and $12.14 \mathrm{ppm}$ are observed for two $\mathrm{NH}$ protons, a singlet for 9.82 ppm for $\mathrm{OH}$ proton, peaks for five aromatic protons are observed at 7.11-7.81 ppm, singlet at 4.53 ppm for $-\mathrm{CH}$ proton and a singlet for $-\mathrm{CH}_{3}$ group is observed at $1.78 \mathrm{ppm}$. Spectral data of compound $\mathbf{2 a}$ fully supports the structure assigned to it.Similarly, other pyrazolo-1,4dihydropyridine derivatives $\mathbf{2 b - n}$ have been synthesised from pyranopyrazoles 1b-nin the mixture of acetic acid and sulphuric acid. The results are summarized in Table 2. 
Table 2. Synthesis of pyrazolo-1,4-dihydropyridines from pyranopyrazoles.

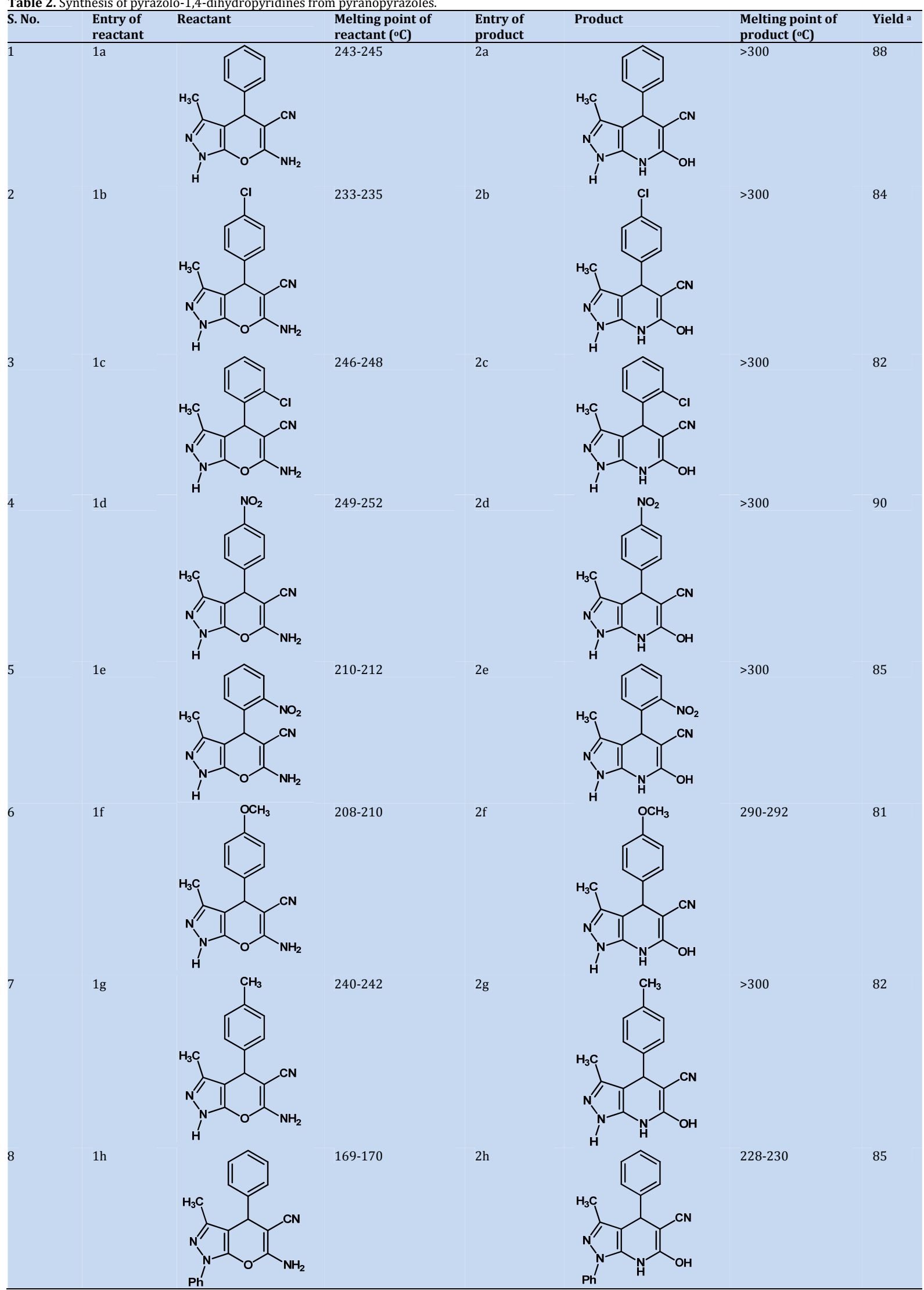

a Yield refer to combined amounts of different crops. 


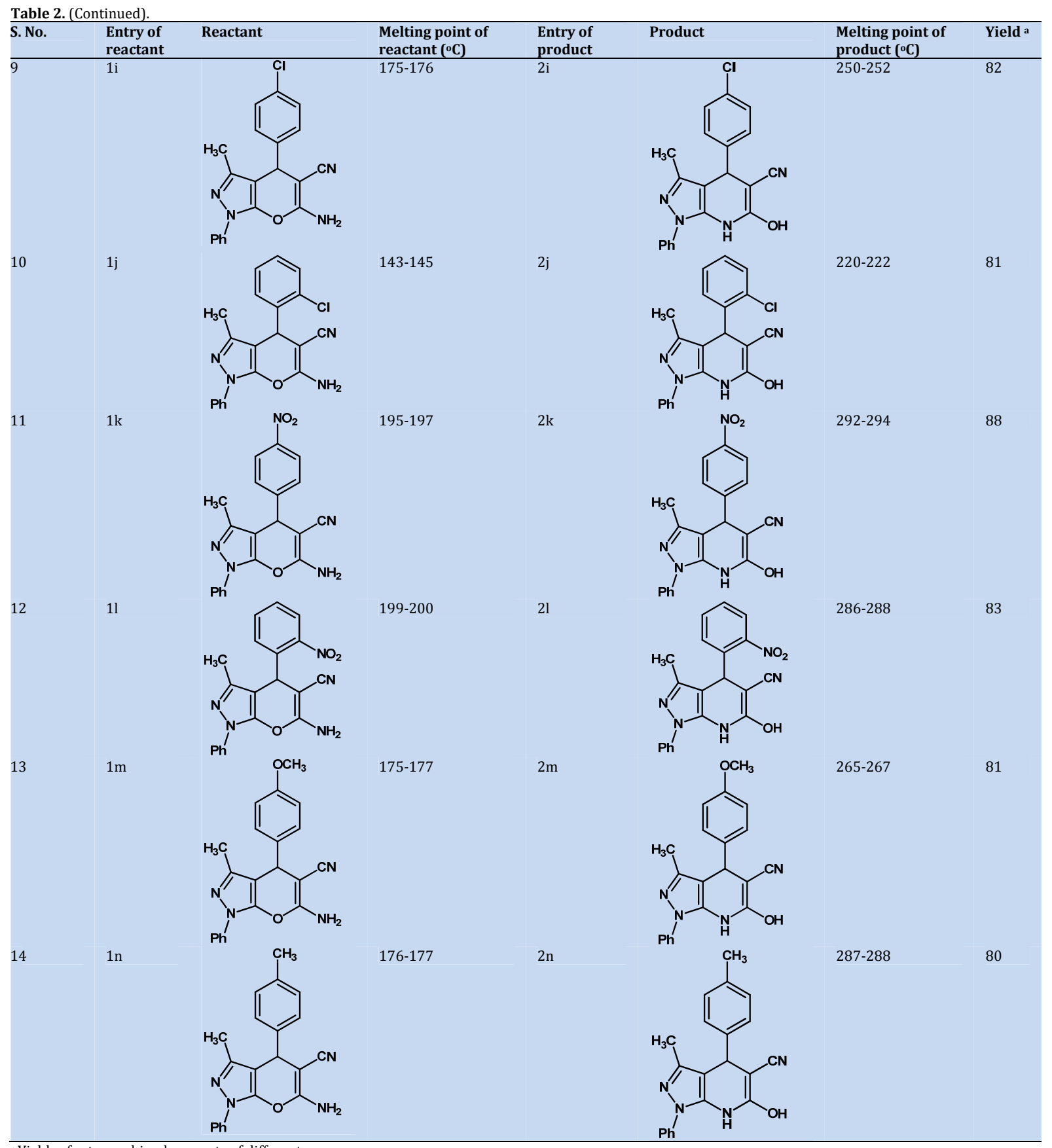

a Yield refer to combined amounts of different crops.

Reactions proceed smoothly with pyranopyrazoles carrying electron withdrawing as well as electron donating substituent's (Table 2). Efficacy of this method is fairly general and affords the resultant products in excellent yield (80-90\%) and products are obtained by simple work up.

Attempt have been made to explain the plausible mechanism of the pyrazolo-1,4-dihydropyridine molecules (Scheme 3). In the acidic medium opening of pyran ring 1 occur via addition of $\mathrm{H}^{+}$ion to give compound 4 which upon tautomerism produced $\mathbf{5}$, as C-C single bond rotation is possible in structure $\mathbf{5}$, on rotating produced $\mathbf{6}$. As we know $\mathrm{C}=0$ bond length is smaller than $\mathrm{C}-\mathrm{NH}_{2}$, then the closing from $\mathrm{NH}_{2}$ terminal is occurred to yield 7. Further in acidic medium the keto form $\mathbf{8}$ is converted into more stable enol form $\mathbf{2}$ supported by the presence of absorption peak around 3550 $\mathrm{cm}^{-1}$ in IR and peak at around $\delta 10 \mathrm{ppm}$ in ${ }^{1} \mathrm{H}$ NMR spectroscopy shows the presence of $\mathrm{OH}$ group and absence of peak $1650-1750 \mathrm{~cm}^{-1}$ confirm that enol form exist.

\section{Conclusion}

The present procedure is an effective method for production of pyrazolo-1,4-dihydropyridine, from easily obtainable initiating materials, in a single step with inherent flexibility and diversity. This method was efficacious to reduce labor, cost, waste production and also devoid of harsh reaction conditions. The target compounds were obtained in an acceptable yield with simple recrystallization as a purification step. 

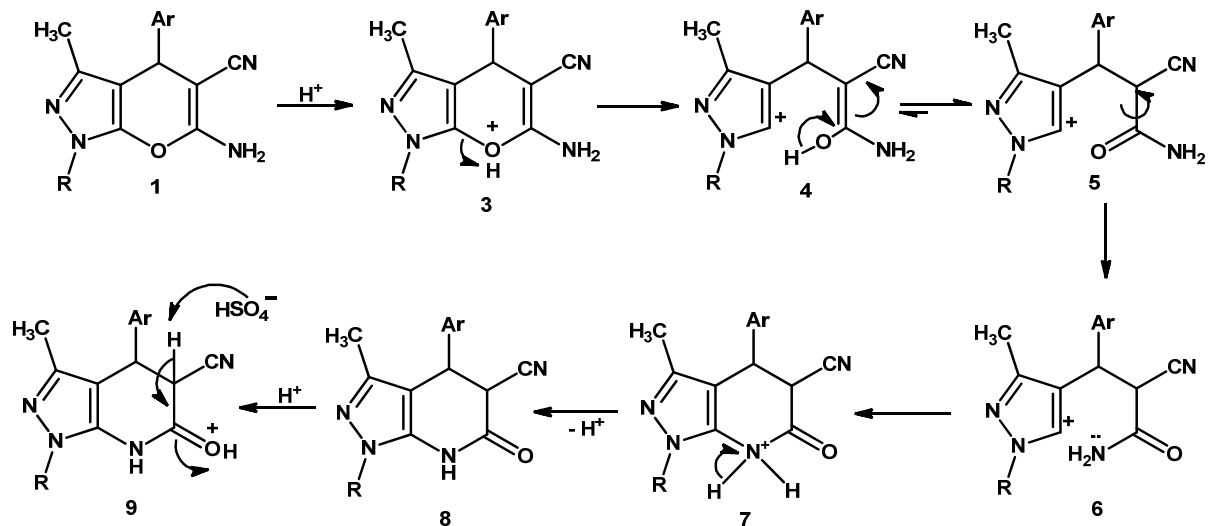<smiles>[R]n1nc(C)c2c1NC(O)=C(C#N)C2[Al]CC#C</smiles>

\section{Acknowledgements}

The authors thank Maharishi Markandeshwar University, Mullana, Haryana, India for the financial support and Harvinder Singh Sohal and Arun Goyal also thank Mr. Vikas Pahwa for the liberal support.

\section{References}

[1]. Hantzsch, A. Jusfus Liebigs Ann. Chem. 1882, 215, 1-82

[2]. Hutton, R. F.; Westheimer, F. H. Tetrahedron 1958, 3, 73-74.

[3]. Rahway, N. J. The Merck Index, 12 $2^{\text {th }}$ edition, Merck Research Laboratories, 1996.

[4]. Bostrom, S. L.; Ljung, B.; Mardh, S.; Forsen, S.; Thulin, E. Nature 1981, 292, 777-778.

[5]. Iwanami, M.; Shibanuma, T.; Fujimoto, M.; Kawai, R.; Tamazawa, K.; Takenaka, T.; Takahashi, K.; Murakami, M. Chem. Pharm. Bull. 1979, 27, 1426-1440.

[6]. Arrowsmith, J. E.; Campbell, S. F.; Cross, P. E.; Stubbs, J. K.; Burges, R. A.; Gardiner, D. G.; Blackburn, K. J. J. Med. Chem. 1986, 29, 1696-1702.

[7]. Goldmann, S.; Stoltefuss, J. Angew. Chem. Int. Ed. Engl. 1991, 30, 1559 1578.

[8]. Sunkel, C. E.; de Casa-Juana, M. F.; Santos, L.; Gomez, M. M.; Villarroya, M.; Gonzalez-Morales, M. A.; Priego, J. G.; Ortega, M. P. J. Med. Chem. 1990, 33, 3205-3210.

[9]. Rovnyak, G. C.; Kimball, S. D.; Beyer, B.; Cucinotta, G.; DiMarco, J. D.; Gougoutas, J.; Hedberg, A.; Malley, M.; McCarthy, J. P.; Zhang, R.; Moreland, S. J. Med. Chem. 1995, 38, 119-129.

[10]. Archibald, J. L.; Bradley, G.; Opalko, A.; Ward, T. J.; White, J. C.; Ennis, C.; Shapperson, N. B. J. Med. Chem. 1990, 33, 646-652.

[11]. Chioua, M.; Samadi, A.; Soriano, E.; Lozach, 0.; Meijer, L.; MarcoContelles, J. Bioorg. Med. Chem. Lett. 2009, 19, 4566-4569.

[12]. Svetlik, J.; Veizerova, L.; Mayer, T. U.; Catarinella M. Bioorg. Med. Chem. Lett. 2010, 20, 4073-4076.

[13]. Chebanov, V. A.; Sakhno, Y. I.; Desenko, S. M.; Chernenko, V. N. Musatov, V. I.; Shishkina, S. V.; Shishkin, O. V.; Kappe, C. O. Tetrahedron 2007, 63, 1229-1242.

[14]. Wang, S. L.; Liu, Y. P.; Xu, B. H.; Wang, X. H.; Jiang, B.; Tu, S. J. Tetrahedron 2011, 67, 9417-9425.

[15]. Wu, L. Q.; Dong, R. Y.; Yang, C. G.; Yan, F. L. J. Chin. Chem. Soc. 2010, 57, 19-23.

[16]. Sohal, H. S.; Goyal, A.; Sharma, R.; Khare, R.; Kumar, S. Eur. J. Chem. 2013, 4, 450-453.

[17]. Sohal, H. S.; Goyal, A.; Sharma, R.; Khare, R.; Kumar, S. Curr. Trends Biotec. Chem. Res. 2013, 3, 12-16.

[18]. Kumar, S.; Goyal, A.; Sohal, H. S.; Kumar, S. Chem. Sci. Trans. 2013, 2, $1459-1465$ 